\title{
A Physical Implementation of the Self-reconfiguring Crystalline Robot
}

\author{
Daniela Rus Marsette Vona \\ Department of Computer Science \\ Dartmouth College \\ Hanover, NH 03755 \\ rus@cs.dartmouth.edu,vona@ai.mit.edu
}

\begin{abstract}
We discuss a physical implementation of the Crystalline robot system. Crystalline robots consist of modules that can aggregate together to form distributed robot systems. Crystalline modules are actuated by expanding and contracting each unit. This actuation mechanism permits automated shape metamorphosis. We describe the Crystalline module concept and a physical implementation of a robot system with ten units. We describe experiments with this robot.
\end{abstract}

\section{Introduction}

Self-reconfiguring robots have the ability to adapt to the operating environment and the required functionality by changing shape. They consist of a set of identical robotic modules that can autonomously and dynamically change their aggregate geometric structure to suit different locomotion, manipulation, and sensing tasks. A primary design goal for a selfreconfiguring robot is to allow the robot to assume any geometric shape. This goal is different from that of other types of shape-changing robots, which may only take one of a small number of shapes.

Self-reconfiguring robot systems may be heterogeneous or homogeneous. In a heterogeneous system, some modules are different from the others. In a homogeneous system all the modules are identical.

In our previous work $[4,5,8]$ we discuss a homogeneous robot system based on a small and simple module we call a Robotic Molecule. This module has already been prototyped. Our experiments demonstrate that it is capable of self-reconfiguration in three dimensions. We have also demonstrated how systems composed of robotic Molecules can use selfreconfiguration to increase their locomotive versatility $[6,7]$. The robotic Molecule uses 4 rotational degrees of freedom to accomplish motion relative to a structure that consists of identical modules.
In this paper we propose a different approach to homogeneous self-reconfiguring robot systems. This approach uses a module called a Crystalline Atom inspired by muscles and amoebas, and is actuated by expansion and contraction. By expanding and contracting the neighbors in a connected structure, an individual module can be moved in general ways relative to the entire structure. Such a movement is illustrated in Figure 3, which shows three snapshots from a simulation of a four-unit robot. This basic operation allows a Crystalline robot system to realize a wide range of geometries; for example Figure 2 shows snapshots from a simulation in which a dog-shaped object transforms itself into a couch-shaped object.

The actuation scheme of the Atom leads to new algorithms for global self-reconfiguration planning. In our previous ICRA paper [17] we presented the concept of a Crystalline module and described a planner for Crystalline robots which we used to generate simulations of self-reconfiguring robots. In this paper we describe a physical implementation of Crystalline Atoms, a robot system composed of 10 Crystalline Atoms, and some experiments with this system. The Crystalline Atom (see Figure 5) has square (cubic in 3D) shape with connectors to other modules in the middle of each face. It is activated by three binary actuators, one to permit the side length of the square to shrink and expand and two to make or break connections to other Atoms. This actuation scheme allows an individual module to relocate to arbitrary positions on the surface of a structure of modules in constant time. Previous systems necessitate linear time in the number of modules on the surface $[23,14,13,6,7]$, because motion from some point $A$ to another point $B$ requires traversing a path between $A$ and $B$ on the surface of the structure. The expansion/contraction actuation of the Crystalline Atom allows a module to 

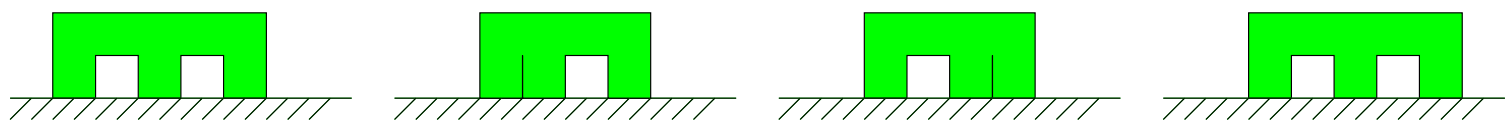

Figure 1: This figure demonstrates using shape metamorphosis for locomotion. A statically stable gait is used to translate the robot from left to right.
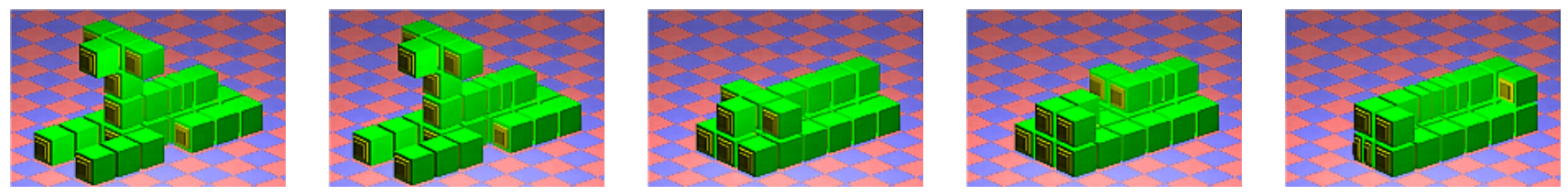

Figure 2: Five snapshots from a simulation using Crystalline robots. The initial configuration (on the left) is a dog-shaped object. The final configuration (on the right) is a couch-shaped object. The middle images show intermediate steps in the transformation from dog to couch. The planning for this transformation was done manually. Note that some Atoms are left in a compressed state so that the volume of the final shape is less than the volume of the initial shape.

relocate from $A$ to $B$ by traveling through the volume of the structure and permits an $O(n)$ reduction in the complexity of planning, as shown in [17]. Our planner, which is called melt-grow ${ }^{1}$ runs in $O\left(n^{2}\right)$ time, where $n$ is the number of modules in the Crystal. Thus, we argue that Crystal Robots provide a simple and effective approach to self-reconfiguration.

In our lab, we have built a self-reconfigurable robot system consisting of 10 Crystalline Atoms. In this paper we report on experiments that use selfreconfiguring Crystalline Atoms for locomotion and shape metamorphosis.

\section{Related work}

Several other groups have done pioneering work related to self-reconfiguring robots. In [1], Fukuda et al propose a cellular robotic system to coordinate a set of specialized modules. Several specialized modules and ways of composing them were proposed. In [21] Yim studies multiple modes of locomotion that are achieved physically by manually composing a few basic elements in different ways. This work also presents extensive examples of locomotion and self-reconfiguration in simulation. In [22], Yim proposes a dodecahedron-based module capable of self-reconfiguration. In $[9,23,20,10]$, Murata et al consider a system of modules called Fracta that can

\footnotetext{
${ }^{1}$ This name reflects the functionality of the planner which operates in two stages: first a shape $S_{1}$ is "melted" into an intermediate shape $I$, from which the desired shape $S_{2}$ is grown. See [17] for details.
}

achieve planar motion by walking over one another. The reconfiguration motion is actuated by varying the polarity of electromagnets that are embedded in each module. In [11] this same group describes a generalization of the Fracta system capable of three-dimensional motion. In [14, 13] Chirikjian et al describe metamorphic robots that can aggregate as two-dimensional structures with varying geometry. The modules are deformable hexagons. This work also examines theoretical bounds for planning the self-reconfiguring motion of such modules. In [8] we have shown a constanttime reduction between robotic molecule structures our group has designed to support self-reconfiguration $[6,7]$ and metamorphic robots [14].

The robot proposed in this paper is different than the previously proposed modules in its actuation capabilities, which lead to new types of self-reconfiguration planning algorithms. The high-level idea of a shrinkable module that can be a cell in a reconfigurable system has been presented by Tanie et al as the patent [19].

\section{The Crystalline Module}

\subsection{Concept}

The Crystalline module is a mechanism that has some of the motive properties of muscles, that can be closely packed in 3D space, and that can attach itself to similar units. We chose a design based on cubes with connectors to other modules in the middle of each face. The idea is to build a cube that can contract by 

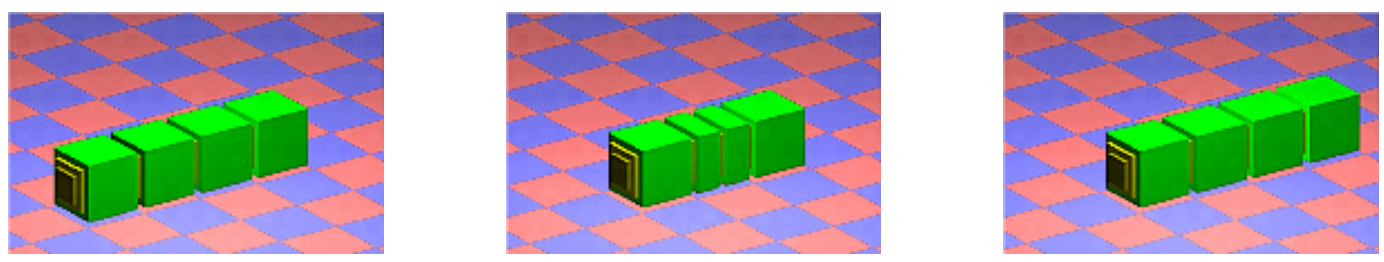

Figure 3: Three snapshots from a simulation of locomotion using Crystalline robots. The left image shows the initial state. The middle image shows the robot after shrinking two modules in the direction of motion. The right image shows the robot after relaxing the shrunk modules in the direction of motion. Notice that the entire structure moved forward one unit, in an inchworm-like fashion. In this simulation the robot is assumed to be walking on a substrate of similar modules, but not all locomotion algorithms require such a substrate.

a factor of two and expand to the original size (see Figures 3 and 2). We wish to effect compression along all three principal directions (e.g., $x, y, z)$ individually or in parallel. We call the module an Atom, and each connector a bond. Figure 4 shows a design for the mechanics of a two-dimensional (square rather than cubic) implementation of the Atom and Figure 5 shows the physical prototype. We use complimentary rack and pinion mechanisms to implement the contraction and expansion actuation for the two-dimensional prototype, as shown in Figure 7. In three dimensions, the rack and pinion mechanisms could be replaced with lead screws. Because squares and cubes are highly regular, most planning algorithms developed in two dimensions can be easily generalized to three. Similarly, algorithms developed in three dimensions can be easily specialized to two dimensional structures.

\subsection{A Physical Implementation}

Crystalline Atomic modules can be constructed in both two and three dimensions. In two dimensions, Atoms are square; in three dimensions they are cubic. In this section we describe the two-dimensional version of the module we constructed.

The two-dimensional version of the Crystalline Atomic module (see Figure 5) was created based on the CAD designs shown in Figure 4. The module has an expansion/contraction ratio of 2 . In this implementation, all four Atom faces are tied together and actuated by a single motor, i.e. they must all be simultaneously fully extended or fully contracted ${ }^{2}$. Each face of the module contains part of a connection mechanism. Two out of the four faces ${ }^{3}$ have active connection mechanisms (see Figure 9). The other two faces

\footnotetext{
${ }^{2}$ An implementation with independently controlled faces would be only slightly more versatile.

${ }^{3}$ The active connection mechanisms are situated on adjacent faces, which allows any lattice of Crystalline Atoms to be fully connected.
}
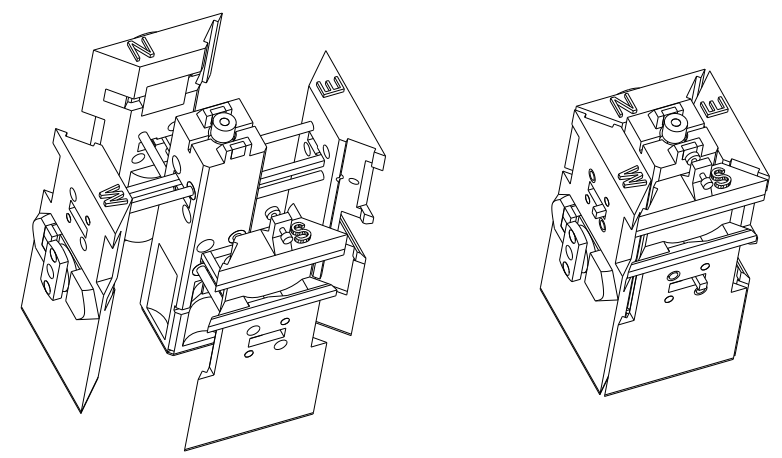

Figure 4: The mechanics of a 2D Atom actuated by complimentary rack-and-pinion mechanisms. The Atom is 4 inches tall (not including electronics, which are not shown, and which increase the height to 7 inches). When expanded (left), the Atom occupies a 4 inch square; when contracted (right) the Atom occupies a 2 inch square.

have passive connection slots (see Figure 8). Together, these provide a key-and-lock system for forming rigid connections with adjacent modules. Thus, the entire unit can be realized with three degrees of freedom: one to expand/contract the faces of the Atom, and two for the active connectors. All three degrees of freedom can be implemented with binary actuators. Since Atoms are not designed to rotate relative to one another, the use of two rather than four connectivity degrees of freedom leads to no mechanical limitations. Every inter-Atomic interface of a structure will have one active connection mechanism, as illustrated in Figure 6 . The module has on-board electronics and five $3 \mathrm{~V} 2 / 3 \mathrm{~A}$ size Lithium batteries, so that it can function untethered. 


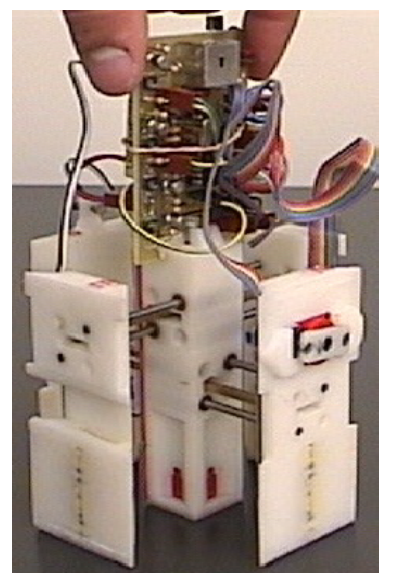

Figure 5: The physical prototype for the Crystalline Atom.

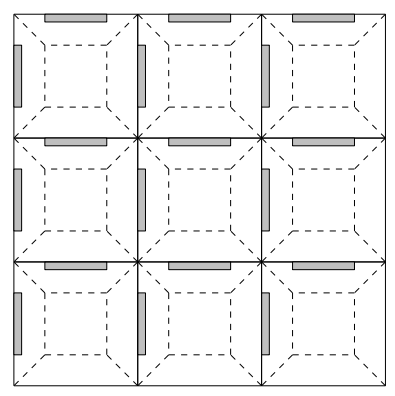

Figure 6: A schematic tiling of 9 compressed Atoms. Note that every inter-Atomic interface contains exactly one active connection mechanism (dark rectangles).

An alternative design would be to allow each face to be fully extended or fully contracted independent of the other faces. This approach would require three additional degrees of freedom and would result in a higher degree of controllability. However, the versatility of such systems with respect to two-dimensional self-reconfiguration is not significantly greater than our proposed more minimalist design.

The expansion/contraction degree of freedom has been implemented with a rack-and-pinion mechanism (see Figure 7). A vertically mounted pinion at the center of the core mates simultaneously all racks. The racks are rigidly mounted to the rear of each face. Racks from opposing faces are mounted off-center and racks from adjacent faces are staggered vertically so that they do not overlap at the center of the core. The

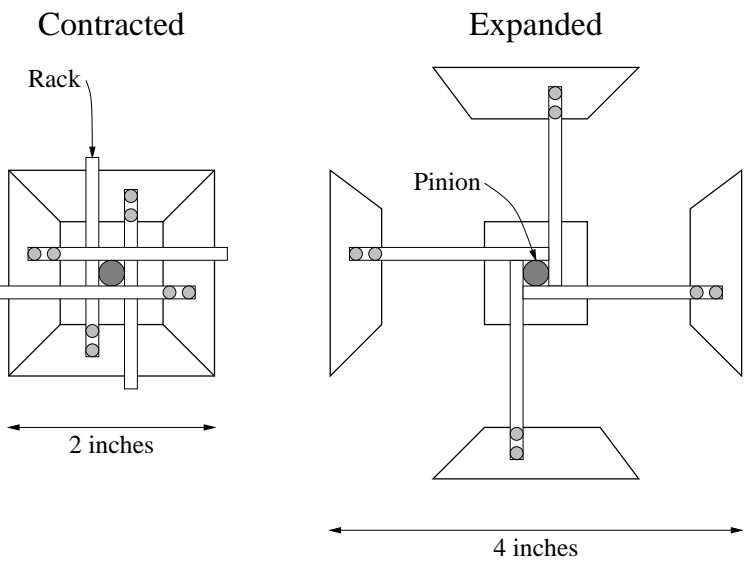

Figure 7: A schematic diagram of the rack-and-pinion mechanism used to actuate Atom expansion and contraction.

pinion is driven by a gear motor. Spinning it in one direction extends the racks, which causes the Atom to expand. Spinning in the other direction retracts the racks which causes the Atom to contract. The motor used for these motions is a Lego toy Mini-Motor, which was selected for its small size (5/8 inch cube), useful torque (2oz-in at $10 \mathrm{rpm}, 12 \mathrm{~V}$ and $80 \mathrm{~mA}$ ), and low-cost (\$11). Two hall-effect sensors serve as bi-level position sensors and are used to control the expansion/contraction movements of the Atoms. One sensor is used to determine if the faces have moved close enough to the core during a contraction movement; the other sensor indicates whether the faces have moved far enough outward during an expansion.

When fully contracted, the Atom is a square with a 2 inch side. When fully expanded, the Atom is a square with a 4 inch side. The height of the Atom is 7 inches and its weight is 12 ounces.

Only lattices whose faces are normal to the $x, y$, and $z$ axes can be created using Crystalline robots. By manipulating the size of the Atom, it is possible to approximate any finite solid shape to an arbitrary precision using Crystalline modules ${ }^{4}$.

The connection mechanisms are based on a channel and key concept (see Figure 9 and 8). The passive face contains a deep horizontal channel on its outer surface. Pockets are built into the upper and lower inside surfaces of this channel at the center of the face. The active face contains a gear-motor (the same model Lego mini-motor that is used to actuate ex-

\footnotetext{
${ }^{4}$ The aliasing error for any shape on a raster display can be arbitrarily reduced by increasing the resolution of the display.
} 
pansion/contraction). A bar (the "key") is attached to the output shaft of the motor. At one angle, the key can slide horizontally through the channel of the passive face without obstruction and the connector is freed. At another angle, the key is rotated so that it extends into the pockets of the passive face and the connector is bonded. Hall-effect sensors are used to determine the position of the connector keys.
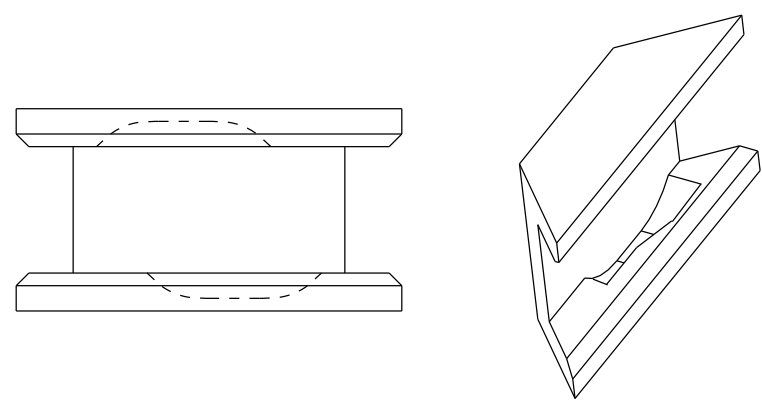

Figure 8: The passive face of the connection mechanism. On the left is a plan view, where the hidden pockets are indicated by dashed lines. On the right is an oblique view, showing the sloped inner surface of the pockets.
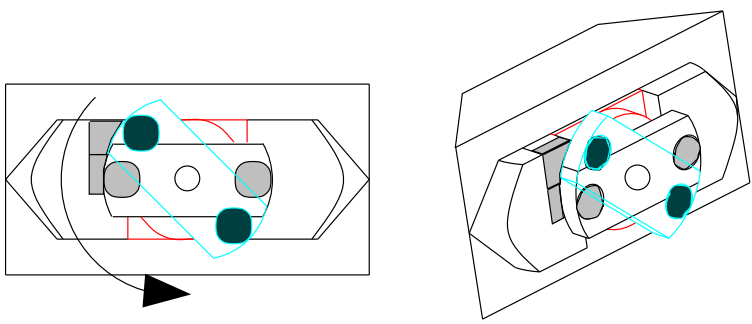

Figure 9: The active face of the connection mechanism. On the left is a plan view, and on the right is an oblique view. The key is shown in the unbonded position (horizontal, dark) as well as in the bonded position (diagonal, light). The key is always rotated in the direction indicated by the arrow. The shaded rectangles are the hall-effect position sensors and the shaded circles are the corresponding magnets.

The connection mechanisms are designed to be misalignment tolerant and correcting. This allows adjacent units which are not precisely aligned to bond, and in the process to become better aligned. Some correction for flexibilities and other inaccuracies in the rest of the mechanics is thereby provided. About +/0.1 inches horizontal misalignment is tolerable in the inter-Atomic plane (i.e. units sliding past each other), and about +0.1 inches misalignment is tolerable in the direction normal to the inter-Atomic plane (units moving away from each other). In-plane misalignment is corrected for by arranging the pockets on the passive connector face to be alternately offset from the midpoint (this is easily seen in the plan view in Figure 8). Misalignment in the direction normal to the inter-Atomic plane is handled by including a slope on the inner surface of the pockets on the passive face (visible in the oblique view in Figure 8).

\subsection{Fabrication}

We have built ten prototype Atoms and an environment of connectors to demonstrate the feasibility of our approach. The parts cost of each prototype Atom is about $\$ 300$, and it takes approximately three days to fabricate and assemble all parts. We generated most parts using an FDM2000 Fused Deposition Modeling Rapid Prototyping machine ${ }^{5}$.

\subsection{On-board Electronics}

Each Atom contains an on-board processor (Atmel AT89C2051 microcontroller), power supply (five $2 / 3$ A Lithium batteries), and support circuitry, which allows both fully untethered and tethered operations. Atoms are connected by a wired serial link to a host computer to download programs. For untethered operations, an experiment specific operating program specified as a state sequence is first downloaded over a tether. When the tether is removed, an on-board IR receiver is used to detect synchronization beacons from the host.

\subsection{Primitive Operations for Crystalline Modules}

Crystalline robot systems are dynamic structures: (1) they can move using sequences of reconfigurations to implement locomotion gaits; and (2) they can undergo shape metamorphosis. The dynamic nature of these systems is supported by the ability of individual modules to move globally relative to the structure.

The basic operations in a Crystalline robot system are:

- (expand <atom>, <dimension>) - expand a compressed Atom in the desired dimension $(x, y$, or $z$ )

- (contract <atom>, <dimension>) - compress an expanded Atom in the desired dimension

\footnotetext{
${ }^{5}$ We used the same fabrication technology described in [6].
} 
- (bond <atom>, <dimension>) - activate one of the Atom's connectors to bond with a neighboring Atom in the structure

- (free <atom>, <dimension>) - deactivate one of the Atom's connectors to break a bond with a neighboring Atom in the structure

Figure 3 illustrates the use of these primitives for generating a linear locomotion algorithm called the inchworm gait for Crystals. The robot consists of four connected Crystalline modules. The modules rest on a substrate of other Crystalline modules ${ }^{6}$. We assume that each module can compress by a factor of 2 . In the first phase of the algorithm, the rightmost module attaches to the substrate and the middle modules compress. This operation causes the leftmost module to advance by one unit (where the unit is denoted by the size of the module). In the second phase of the algorithm the leftmost module makes a connection to the substrate, the rightmost module disconnects and the middle two modules expand. The net effect of this algorithm is a global translation of one unit for the Crystal. It is possible to describe similar algorithms for effecting global translations and 90 degree concave and convex transitions about Crystalline structures.

\section{Experiments with Self-reconfiguring Crystals}

We have constructed ten prototype modules and used them to perform experiments to evaluate the feasibility of using multiple Atoms to demonstrate reconfiguration.

To facilitate experimentation, a row of 8 fixed passive connectors was constructed to simulate the surface of a Crystal. This arrangement not only frees us from having to construct many units at the outset, but it also allows us to perform experiments that are focused narrowly on the specific activities under study. The fixed connectors are placed as they would be for a flat Crystal surface composed of 8 contracted Atoms. In the descriptions that follow, we will refer to two of the prototype Atoms as $\mathbf{a}$ and $\mathbf{b}$, and we will number the fixed connectors $\mathbf{0}-\mathbf{7}$. The North and West faces of $\mathbf{a}$ and $\mathbf{b}$ (those that contain active connection mechanisms) will be referred to as a.n/b.n and a.w/b.w, respectively, and the South and East faces will be similarly named. $\mathbf{a}$ and $\mathbf{b}$ are always oriented so that $\mathbf{a . n}$ and b.n are facing the row of fixed connectors.

The first experiment was designed to determine if an Atom could reliably expand and then connect with

\footnotetext{
${ }^{6}$ Note that the substrate is not necessary for all locomotion gaits.
}

a neighbor. Initially, a was expanded and affixed to $\mathbf{0}$ (at a.n). b was contracted and affixed to $\mathbf{2}$ at (b.n). The following state sequence was then executed on the Atoms, as shown in Figure 10:

\section{1. expand $\mathbf{b}$ \\ 2. connect b.w}

The first experiment yielded positive results. It demonstrated that $\mathbf{b}$ could reliably expand and connect with a in most cases. One situation where it was observed to fail was when an especially low-friction environment surface was used. In this case, step 2 usually does not complete because play in the mechanics allows Atom a to be too easily pushed away from Atom $\mathbf{b}$ as it expands in step 1.

The second experiment was designed to evaluate whether Atoms could work together to effect a reconfiguration. Initially, both $\mathbf{a}$ and $\mathbf{b}$ were contracted. a was connected to $\mathbf{0}$ (at $\mathbf{a} . \mathbf{n}$ ) and $\mathbf{b}$ was connected to $\mathbf{1}$ (at b.n). $\mathbf{a}$ and $\mathbf{b}$ were connected together at $\mathbf{b} . \mathbf{w}$. The Atoms were programmed with state sequences designed to perform a variant of inchworm translation along the fixed connectors:

1. free $\mathbf{b} \cdot \mathbf{n}$ from $\mathbf{1}$
2. expand $\mathbf{a}$
3. expand $\mathbf{b}$
4. connect $\mathbf{b . n}$ to $\mathbf{2}$
5. disconnect a.n from $\mathbf{0}$
6. contract a and $\mathbf{b}$
7. connect a.n to $\mathbf{1}$
8. repeat

This sequence is illustrated in Figure 11, and Figure 12 presents several photographs of the Atom prototype hardware performing the experiment.

In the third experiment, we explored the motion primitives for Crystal modules in a structure constructed out of 10 modules. We created a $3 \times 3$ square structure with an additional Crystal module on its surface. We developed a program that allowed this tenth Crystal module to be propagated across a row in the $3 \times 3$ Crystal. Figure 13 shows snapshots from this experiment. We are currently using the 10 modules to experiment with more extensive shape morphing.

\section{Discussion and Future Work}

We presented the Crystalline Atom and showed experimentally that it can be the basis for homogeneous, unit-modular, self-reconfigurable robot systems. The module is inspired by a muscle-like actuation mechanism. The Atom has 3 DOFs, two that allow it to 

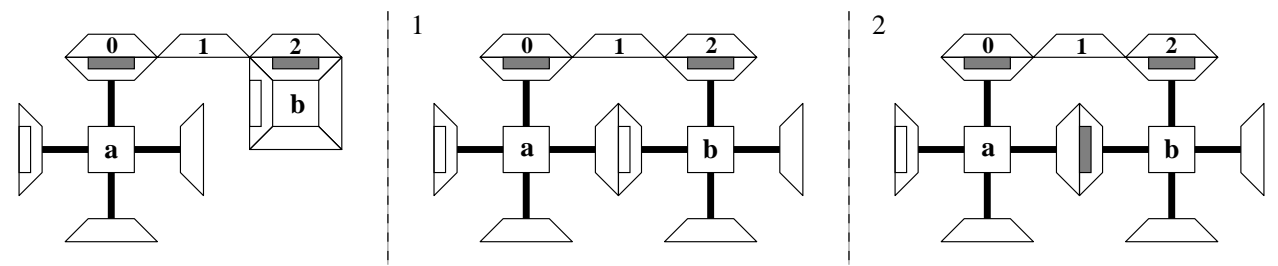

Figure 10: In the first experiment, a was affixed to $\mathbf{0}, \mathbf{b}$ to $\mathbf{2}$. $\mathbf{b}$ was programmed to expand and connect with $\mathbf{a}$.

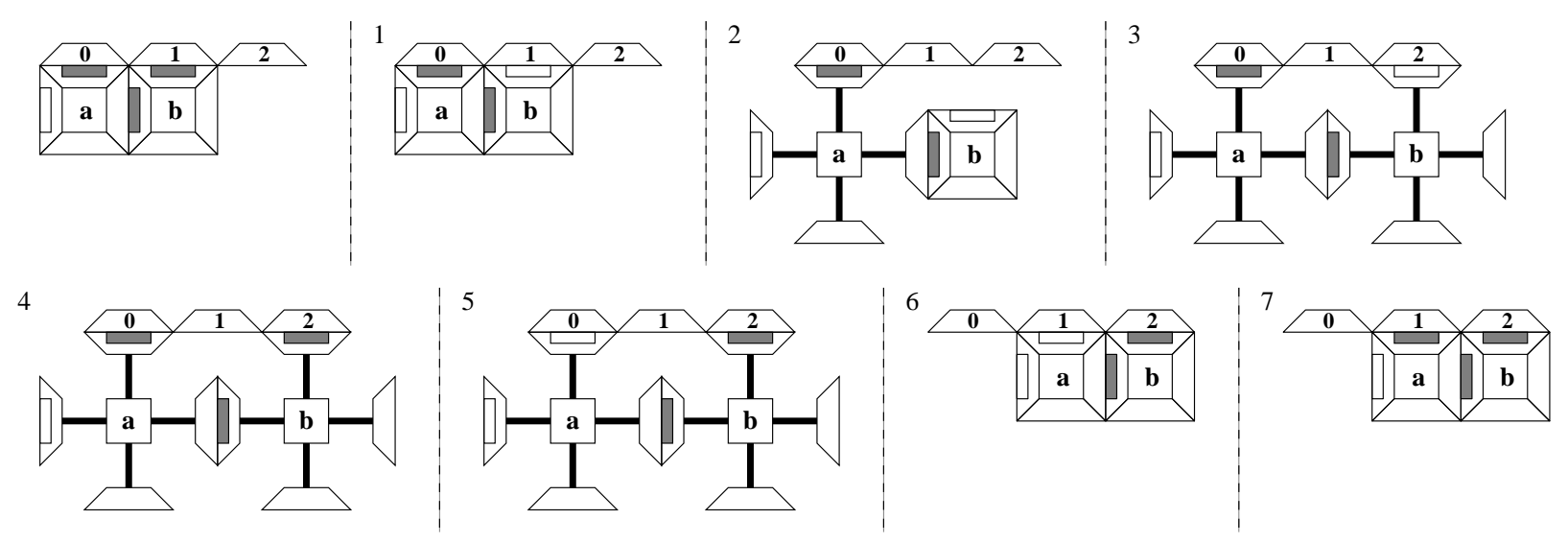

Figure 11: The second experiment tests an inchworm propagation algorithm.

make and break connections with identical modules, and one to actuate expansion and contraction. It is capable of expanding and contracting by a factor of two. This actuation mechanism supports very fast algorithms for relocating one module on the surface of a Crystal, which leads to an efficient $O\left(n^{2}\right)$ planner for shape metamorphosis. We have described our first physical prototypes for the Atom and discussed our experimental results.

In the near future we will be focusing our attention on improving the hardware implementation and on developing distributed reconfiguration algorithms.

\section{Acknowledgements}

We are grateful to Laura Ray and Keith Kotay for extensive discussions about the Crystalline module. We are also grateful to Michael Shin for his help in implementing the simulator.

This paper describes research done in the Dartmouth Robotics Laboratory. Support for this work was provided through the NSF CAREER award IRI-9624286, NSF award IRI-9714332, NSF award EIA-9901589, and NSF award IIS-98-18299.

\section{References}

[1] G. Chirikjian, A. Pamecha, and I. Ebert-Uphoff. Evaluating Efficiency of Self-Reconfiguration in a Class of Modular Robots. Journal of Robotic Systems, Vol. 13., No. 5., May 1996.

[2] T. Fukuda and Y. Kawauchi. Cellular robotic system (CEBOT) as one of the realization of self-organizing intelligent universal manipulator. In Proceedings of the IEEE Conference on Robotics and Automation, pp. 662-667, 1990.

[3] G. Hamlin and A. Sanderson. Tetrabot modular robotics: prototype and experiments. In Proceedings of the IEEE/RSJ International Symposium of Robotics Research, pp 390-395, Osaka, Japan, 1996.

[4] Kazuo Hosokawa, Isao Shimoyama, and Hirofumi Miura. Dynamics of self-assembling systems - analogy with chemical kinetics. Artificial Life, 1(4), 1995.

[5] K. Kotay, D. Rus, M. Vona, and C. McGray. The self-reconfigurable robotic molecule. In Proceedings of the 1998 International Conference on Robotics and Automation, 1998.

[6] K. Kotay, D. Rus, M. Vona, and C. McGray. The self-reconfiguring robotic molecule: design and con- 

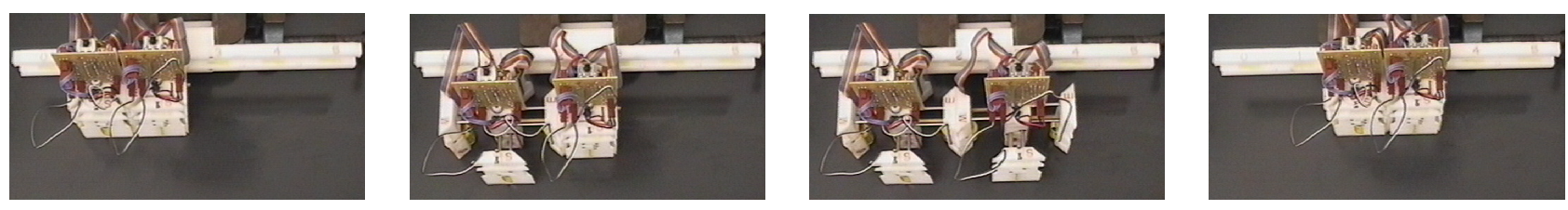

Figure 12: Several snapshots of the Atom prototype hardware performing the inchworm experiment.
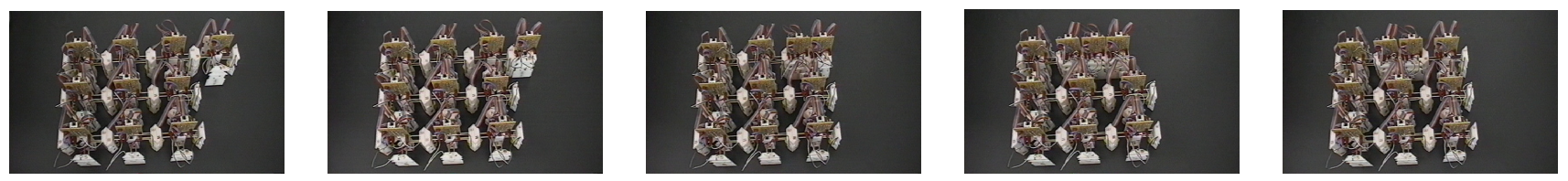

Figure 13: Several snapshots of a 10 module Crystal performing atom relocation by propagation. The left most figure shows a $3 \times 3$ square robot with an additional module in the NE corner; the next three figures show the system after compressing three adjacent modules in sequence, and the last figure shows the final configuration.

trol algorithms. In the 1998 Workshop on Algorithmic Foundations of Robotics, 1998.

[7] K. Kotay and D. Rus. Motion Synthesis for the Selfreconfiguring Robotic Molecule. In Proceedings of the 1998 International Conference on Intelligent Robots and Systems, 1998.

[8] K. Kotay and D. Rus. Locomotion Versatility through Self-reconfiguration In Robotics and Autonomous Systems, 1998 (to appear).

[9] C. McGray and D. Rus. Motion Self-reconfiguring Molecules as 3D Metamorphic Squares In Proceedings of the 1998 International Conference on Intelligent Robots and Systems, 1998.

[10] S. Murata, H. Kurokawa, and Shigeru Kokaji. Selfassembling machine. In Proceedings of the 1994 IEEE International Conference on Robotics and Automation, San Diego, 1994.

[11] S. Murata, H. Kurokawa, K. Tomita, and Shigeru Kokaji. Self-assembling method for mechanical structure. In Artif. Life Robotics, 1:111-115, 1997.

[12] S. Murata, H. Kurokawa, E. Yoshida, K. Tomita, and S. Kokaji. A 3-D Self-Reconfigurable Structure. In Proceedings of the 1998 IEEE International Conference on Robotics and Automation, Leuven, 1998.

[13] A. Pamecha, I. Ebert-Uphoff, and G. Chirikjian. Useful Metrics for Modular Robot Motion Planning. IEEE Transactions on Robotics and Automation pp531-545, Vol.13, No.4, August 1997.

[14] C. Paredis and P. Khosla. Design of Modular Fault Tolerant Manipulators. In The First Workshop on the
Algorithmic Foundations of Robotics, eds. K. Goldberg, D. Halperin, J.-C. Latombe, and R. Wilson, pp 371-383, 1995.

[15] D. Rus. Self-Reconfiguring Robots. IEEE Intelligent Systems, 13(4), 2-5, July/August 1998

[16] D. Rus and M. Vona. Self-Reconfiguration Planning with Unit Compressible Modules. Proceedings of the 1999 IEEE International Conference on Robotics and Automation, pp 2513-2520, Detroit, MI, 1999.

[17] D. Rus and M. Vona. A Physical Implementation of the Crystalline Robot. Submitted to the 2000 IEEE International Conference on Robotics and Automation, San Frncisco, CA, 2000.

[18] K. Tanie and H. Maekawa. Self-reconfigurable cellular robotic system. US Patent 5361186, 1993.

[19] K. Tomita, S. Murata, E. Yoshida, H. Kurokawa, and S. Kokaji. Reconfiguration method for a distributed mechanical system. In Distributed Autonomous Robotic Systems 2, pp 17-25, Springer Verlag 1996.

[20] M. Yim. A reconfigurable modular robot with multiple modes of locomotion. In Proceedings of the 1993 JSME Conference on Advanced Mechatronics, Tokyo, Japan 1993.

[21] M. Yim. Polypod II. http://www . parc .xerox.com/spl/members/yim/

[22] E. Yoshida, S. Murata, K. Tomita, H. Kurokawa, and S. Kokaji. Distributed Formation Control of a Modular Mechanical System. In Proceedings of the 1997 International Conference on Intelligent Robots and Systems, 1997. 